\title{
Balancing the Inpatient-Nurse Relationship in Anorexia Care
}

\author{
Kristine Rørtveit1,2 (), Anna-Maria Sikveland 1,2, Britt Saetre Hansen 1,2 (]) \\ ${ }^{1}$ The Nursing and Healthcare Research Group, Stavanger University Hospital, Stavanger, Norway \\ ${ }^{2}$ The Research Group for Life Phenomena and Caring, Faculty of Health Sciences, University of Stavanger, \\ Stavanger, Norway \\ Email:kristine.rortveit@sus.no
}

How to cite this paper: Rørtveit, K., Sikveland, A.-M. and Saetre Hansen, B. (2020) Balancing the Inpatient-Nurse Relationship in Anorexia Care. Open Journal of Nursing, 10, 858-872.

https://doi.org/10.4236/ojn.2020.109059

Received: August 4, 2020

Accepted: September 19, 2020

Published: September 22, 2020

Copyright (C) 2020 by author(s) and Scientific Research Publishing Inc. This work is licensed under the Creative Commons Attribution International License (CC BY 4.0).

http://creativecommons.org/licenses/by/4.0/

\begin{abstract}
Background: Nurses play a central role in the treatment of inpatients with anorexia, where bonding is a key factor. The inpatient-nurse relationship is based on a confident alliance characterized by important factors such as trust, confidentiality, responsiveness, genuineness, and consistency. Aim and Research Question: The aim of this study was to describe patients' in-depth experiences of their relationship with nurses when hospitalized for anorexia. The research question was: How do women hospitalized due to anorexia nervosa experience the nurse-patient relationship? Method: A hermeneutic approach with in-depth interviews was employed. Five women with previous experience of inpatient care for anorexia were interviewed. Findings: The findings summarized how the women experienced the relationship with professional nurses in a ward characterized by a high degree of structure. The main theme Balancing feelings of emotional contradictions in the patientnurse relationship was described by two sub-themes; Opening up for a care relationship in a rigid and structured ward atmosphere and Fluctuating in a paradox of conflicting feelings. Discussion: From the patients' perspective, balancing the patient-nurse relationship when an inpatient involves conflicting emotional challenges. Structured interventions are perceived as effective by the patients, although they may be ambivalent about them. Therefore, a good relationship depends on the establishment of emotional and stable interaction with the nurses. Implications for Nursing Practice: Patients need interventions based on safe and competent quality care. A hermeneutic approach may improve the nurses' ability to meet these patients in their lifeworld and establish a confident relationship. Special education related to anorexia care should be a priority in nursing.
\end{abstract}

\section{Keywords}

Anorexia Nervosa, Eating Disorders, Hermeneutics, Nurse-Patient 
Relationship

\section{Introduction}

Anorexia is defined by the International Classification of Diseases and related health problems (ICD-10) as a "disorder characterized by deliberate weight loss, induced and maintained by the patient (...). Fear of obesity and chubby figure is intrusive and oversized" [1]. Patients suffering from anorexia often have symptoms of malnutrition, rigid eating habits, and excessive exercise. There is no single explanation of what causes eating disorders, but psychological, biological, and cultural factors contribute to and influence disease development [2]. Most research has focused on anorexia among teenagers; however $40 \%$ of those affected are adults, who may experience different treatment challenges [3].

\subsection{Background}

Previous research indicates that in the treatment of anorexia patients the quality of the relationship is crucial for the outcome [3]. Nurses play a central role in the treatment of inpatients with anorexia, where bonding is a key factor [4]. A therapeutic relationship is characterized by empathy, positive regard, and acceptance as well as non-judgmental responses. Other characteristics such as trust, confidentiality, responsiveness, genuineness, and consistency have also been highlighted [5]. The importance of a confident alliance and the nursing characteristics that enhance the quality of the relationship have been described [6]. Awareness of transference and countertransference when dealing with anorexia patients is important [7], as is the creation of a trusting relationship [8]. Research confirms that when trying to describe the quality of the relationship between patient and nurse, the patient's experience often differs from that of the nurse [9]. The nurse's role of monitoring treatment can lead to anxiety and stress for the patient, which constitutes a particular challenge in the development of a good relationship [4].

Increased knowledge of factors that require attention is essential for the development of quality care for anorexia patients.

\subsection{Aims and Research Questions}

The aim of this study was to describe patients' in-depth experiences of their relationship with nurses when hospitalized for anorexia. The research question was: How do women hospitalized due to anorexia nervosa experience the nurse-patient relationship?

\section{Methods}

\section{Study Design and Methods}

The design was explorative and descriptive. We used a hermeneutic approach 
[10] and collected data by means of in-depth interviews. Hermeneutics refers to the theory and practice of interpretation in order to gain a deeper understanding of human experiences. The hermeneutic circle is often used to describe how understanding and meaning are developed. Understanding evolves in a circular process of moving back and forth between the whole and the parts. The parts are thereafter assembled into a new whole with an enhanced level of understanding. As the circle is further developed new understanding and knowledge are continuously created [11].

The data were based on in-depth interviews in the form of a dialogue between the second author (AS) and five women with previous experience of inpatient care for anorexia nervosa, which enabled investigation of the women's experience of meaning and understanding [12]. The in-depth interviews were audiotaped and transcribed verbatim, after which qualitative content analysis [13] was used to reveal the meaning of the data.

\section{1) The Researchers' Pre-Understanding}

Nursing practice has been part of the three authors' lives for many years and therefore their pre-understanding was influenced by nursing experiences. Preunderstanding allows us to access the world so that we can understand, interpret, and understand again [10]. The first author (KR) is an associate professor who works as a research advisor and as a clinical nurse in a psychiatric outpatient ward. The second author (AMS) has been employed in the clinical field for several years, but was a full time master student when she performed this study. The third author (BSH) is a professor and clinical nurse in an intensive care unit. In their clinical practice the three authors have encountered patients with anorexia in different contexts. They tried to maintain an objective perspective on the analysis that was only colored by their clinical and professional knowledge of the area. From the start the research process was characterized by this perspective, with special focus on potential biases related to the research questions, the analysis of the findings, the discussion, and implications for nursing.

\section{2) Participants}

A convenient sample was used. The data was collected by means of qualitative in-depth interviews as a part of a master degree project for the second author (AMS) in 2013. Initially eight women were invited to participate, but two withdrew for practical reasons and one was hospitalized before the interview was conducted. Five women aged between 22 and 58 years who had a history of hospitalization due to anorexia were finally interviewed. The number of informants depended on findings and saturation, as well as access to informants. The interviews provided rich and emotional descriptions of their in-patient experiences as the five participants shared willingly. The data was therefore deemed to meet the criteria for saturation.

As part of the recruitment strategy the second author (AMS) sent a letter to several outpatient clinics and eating disorder interest groups on the west coast of 
Norway with information about the project and an invitation to participate. Women who had been told about the project by others and were interested also received an invitation to participate. The interviews were conducted using open ended questions and focused on the women's experiences of their own treatment process, the nurse-patient relationship, and how a good relationship can be developed in parallel with following a treatment model. The interviews were facilitated, digitally recorded and transcribed verbatim by the second author (AMS). Later, the data was reanalyzed, bearing the research question of the current study in mind.

\section{3) Analysis}

The data analysis was guided by content analysis [13], as the qualitative method should capture both the manifest and latent meaning of the text. The data were read and re-read several times in order to grasp their meaning, after which codes were developed and the second author (AMS) organized the text into sub-themes. In this step of the analysis, all authors discussed the understanding several times, always keeping the research question and meaning units in mind and remaining close to the original transcripts. Finally, the sub-themes and main theme were analyzed by the first author (KR), and a comprehensive understanding was formulated into one main-theme. All authors validated the sub-themes, the understanding of which was interpreted into the main-theme. The method complements the hermeneutic approach as it focuses on the dialectical process of moving between the parts and the whole [13].

\section{4) Ethical Considerations}

Ethical guidelines were followed and the research process was guided by ethical principles to ensure that each patient's dignity, integrity, self-determination, and confidentiality were protected [14]. The requirement of voluntariness was fulfilled through informed consent. The project also received approval from the Regional Ethics Committee (REC, registration number 1862), as well as from the Data Protection Officer at Stavanger University Hospital. Data were stored and handled by the first author (KR) according to regulations. The material was de-identified and the recordings deleted after transcription in accordance with ethical research guidelines. Information was rendered anonymous before presentation of the study by using fake names for the women. The women agreed to participate after receiving information about the study, their right to withdraw at any time and how the data would be stored. The five women signed the written consent form.

\section{Findings}

Five women with anorexia aged between 22 and 58 years were recruited. In accordance with the inclusion criteria, none of them were hospitalized at the time of the interviews. Health status and attitude to admission were not examined. Through working with the in-depth data from the five interviews, a theme emerged that described experiences of the relationship with professional nurses 
in a ward characterized by a high degree of structure. The main theme and sub-themes were framed within the context of anorexia care. The main theme was formulated as follows: Balancing feelings of emotional contradictions in the patient-nurse relationship when hospitalized for anorexia. The main theme was based on two sub-themes; Opening up for a care relationship in a rigid and structured ward atmosphere and Fluctuating in a paradox of conflicting feelings. A summary of the findings is presented in Table 1.

\subsection{Balancing Feelings of Emotional Contradictions in the Patient-Nurse Relationship}

Being an inpatient suffering from anorexia led to mental battles. This was illuminated when the women reflected on the process of becoming involved with nurses and inpatient care structures, while at the same time being emotionally challenged by the stressors due to a rigid structure that targeted eating conditions. The women reported different levels of conflict with the inner and outer world, which were affected by this process. These conflicts were interpreted and understood as balancing feelings. The main theme was divided into two themes that described the challenges of entering a nursing care relationship and the challenges of coping with conflicting feelings.

\subsubsection{Opening Up for a Care Relationship in a Rigid and Structured Ward Atmosphere}

All the women had received different structured anorexia treatment interventions and claimed that the ward atmosphere, including firm rules and structure, was important for their progress towards better health. Their experiences of types of intervention, how the intervention was carried out, and its influence on the quality of care varied. However, it seemed as if the "what" (what type of intervention) was less important than the "how" (how the intervention was presented and followed up).

\section{1) Increasing Confidence}

A care relationship that facilitated feelings of safety and confidence was essential when adhering to detailed rules. One woman reflected on how she had been allowed time to settle in and feel safe before having to follow the rules; it made her feel prepared and more confident.

Table 1. Overview of the findings.

\begin{tabular}{ccc}
\hline \multirow{2}{*}{ Themes } & $\begin{array}{r}\text { Balancing feelings of emotional contradictions } \\
\text { in the patient-nurse relationship }\end{array}$ \\
\cline { 2 - 3 } & $\begin{array}{c}\text { Opening up for a care relationship in a } \\
\text { rigid and structured ward atmosphere }\end{array}$ & $\begin{array}{c}\text { Fluctuating in a paradox of } \\
\text { conflicting feelings }\end{array}$ \\
Sub-themes & $\begin{array}{c}\text { Increasing confidence } \\
\text { Needing predictability } \\
\text { Seeking individualized care }\end{array}$ & $\begin{array}{c}\text { Sensing availability } \\
\text { Lenging for caring and responsive nurses }\end{array}$ \\
\hline
\end{tabular}


...the treatment that I received was very detailed, (...) I think there was no alternative, it was best for me. Because it was so incredibly detailed, it was not possible to fool around.

However, too much focus on weight gain and rules could reduce the women's confidence in the relationship. Weight gain was perceived as easier to deal with if simultaneously given the opportunity to be active. Two of the women expressed the sense of being in prison or being a caged lion due to the fact that facilitating confidence in the patients was not prioritized. One drew similarities to a machine:

I probably felt more like a machine that must be fixed than a human, they put food in the tank and you were punished if you did not gain enough weight. Then you had to eat more and the rules became stricter.

One woman experienced that too much focus on rules resulted in less help to cope with difficult emotions. She felt that the treatment made her feel even more ill as she had limited opportunities to talk about her struggle with the emotions behind her anorexia. The women wanted to work with mealtime challenges, weight gain and difficult emotions in parallel.

...because when you' re just talking about food and weight (...) at the same time as I ate more, the trauma became worse and worse. In a way, I did not manage to cope with it.

\section{2) Needing Predictability}

Predictability was a key to feelings of trust, developing a caring relationship, and seemed to help the women to deal with their own feelings.

Predictability and not being left alone were essential. For instance, knowing that one could have a daily conversation with the nurse, being informed about one's individual treatment plan, and who one's contact person is every day were highlighted. Such predictability was vital for opening up to receiving care and support.

What I thought was hard was that I never knew before the morning meeting if I would get an opportunity to talk to someone that day and that was very difficult. It's like that predictability... if I had known that "you can talk to me every day the first week' (...) that there was very close monitoring in the beginning, that you were not left by yourself.

A high degree of monitoring the adherence to the rules and structure, which was used as a form of control, interrupted the development of a caring relationship but could be a necessary path to better health when carried out in a predictable and caring way.

I had a nurse who was very confident and strict, and I was really scared of her at first (...). She was very smart. I eventually experienced that I needed the strictness, but it was pretty tough. She was very smart and clear. I re- 
member her very well and I liked her.

\section{3) Seeking Individualized Care}

Individualized care was essential. A number of the women expressed that conversations should be adapted to the patient's perception of her own individual needs and condition. The benefit of conversations depended on meeting each woman in her present state and giving her an opportunity to express her needs. One woman stated that it was not only the level of care and treatment that was essential for the outcome, but that her own attitude to accepting treatment also influenced how she made use of it.

But it is partly up to yourself, how much effort you make to adhere to the treatment because I cheated with the rules, and probably cheated myself too.

The sense of a caring environment was described as necessary although not taken for granted; as such feelings were dependent on issues related to their experiences of quality care. The feeling of receiving individual care seemed to be very important.

I think it is very important to have treatment that is adapted to the individual. They have a method that they apply to everyone but people differ enormously. They allowed crocheting for a maximum of 15 minutes. Crocheting helped me! So why was I not allowed to do it for more than $15 \mathrm{mi}-$ nutes?

In summary, factors concerning quality care such as confidence, predictability, and individualized care were important for the women when opening up to the strict ward atmosphere, represented by different structured anorexia treatment interventions.

\subsubsection{Fluctuating in a Paradox of Conflicting Feelings}

All the women described struggling with conflicting feelings and trying to contain them during the treatment period. The ward atmosphere was crucial for ensuring a good foundation for the treatment.

\section{1) Being Stressed by the Atmosphere}

The women described that in the beginning the atmosphere could lead to stress. During the initial phase of treatment, some women reported feeling insecure and anxious when following treatment rules. Such feelings hindered a close relationship with the nurses. For one woman fear and vulnerability were the dominant feelings at the beginning. Being afraid and the sense of being alone during treatment made her feel emotionally unwell.

I was generally very anxious. I remember I didn't have the courage to knock on the door and ask for help. (...) I only asked for permission to go out to be alone.

Lack of confidence was associated with the impression that the nurses adopted 
a patronizing attitude, which led to feelings of humiliation. These impressions were strengthened by the daily life at the ward. For instance, one woman stated that the focus on weight made her feel that there was something wrong with her, as she did not consider weight that important. Another participant expressed that when normal behavior was treated as something suspicious, it could provide a basis for new symptoms.

In the unit they said: "What's in your bag?" when I came back, I felt like a naughty child. It was unpleasant. You know, many times I went to my room and cried.

Feeling confident would have helped this woman to achieve the treatment goal in parallel with coping with the strictness that she perceived as challenging. For example, a relaxed social setting after meals together and physical activity felt reassuring and led to a more normal atmosphere. Confidence and support in dealing with weight and appearance were important. However, this could fluctuate and the examples given were often taken from the context of daily life in the ward, as in the following quotation:

I clearly remember episodes in which I was cared for. It made me feel better and helped me to express my feelings.

\section{2) Sensing Availability}

The women described becoming stressed if the nurses were not around when they needed them. One reported that meeting someone to talk to created a desire to do the right thing. The nurses should have time and be available when the patients need them. When they were unavailable, the women described having to deal with stress alone, which hindered the development of a positive relationship. They needed to talk about difficult feelings and when the treatment only focused on weight and food the women felt it was of no benefit.

(...) when you start to eat, feelings you have tried to ignore come back. Feelings you don't manage to cope with. If you have no one to talk to when this happens you might do other stupid things (...)

\section{3) Longing for Caring and Responsive Nurses}

This theme seemed to be the most important as it was often mentioned by the women and included the qualities that enabled nurses to enter into a satisfactory relationship. They stated that a caring relationship increased their ability to benefit from hospitalization and wished for nurses who were: confident, determined, rigorous and clear, able to express understanding, with experience and knowledge of eating disorders, in addition to being consistent, keeping an eye on things, and keeping promises.

I think the nurse I was in contact with most made me very confident. She was clear, really very confident. When we discussed and disagreed she was very rigorous, repeating the same things. I never managed to make her insecure. It made me confident. 
They stated that if the nurses did not trust the patients and were suspicious of their explanations it hindered the development of a caring relationship.

Well I don't think that relationship alone is enough to make you well, they (the nurses) have to know what to do to help. But I think that trust probably has the most... that a good relationship is most important.

One woman stated that a responsive relationship made her able to deal with difficult situations and gave her courage to articulate her needs.

I think it was important, being allowed to express all those things and being met with respect. I felt they confirmed that they had heard it before and others had similar thoughts.

If the nurse showed a lack of understanding about the patient's feelings in relation to treatment, it created uncertainty in the patient and hindered the development of a good relationship. The women indicated that inexperienced graduates, as well as young or insecure nurses had a negative effect on trust and confidence, which made it more difficult to ask for help. A nurse who lacked knowledge and structure made the women feel that they must manage the treatment themselves.

They (nurses with little experience) were more insecure, afraid. They had to check everything and then I sort of lost confidence and faith in them. I felt I had to check out everything myself as well. When they said something I didn't trust them.

The women expressed the importance of a nurse being responsive and caring, while at the same time strict about following up rules. When carrying out specific interventions, the nurse should show respect and interest in understanding the patient's actions and feelings.

(...) to the extent that a caregiver can provide care to the patient, caring is extremely important (...), to me it was important. Nurses who I felt really cared helped me a lot more.

In summary, the conflicting feelings described in this sub-theme were often influenced by the relationship with nurses. The feelings were associated with the nurses' attitudes, availability, responsiveness, carefulness, experience, and trustworthiness.

\section{Discussion}

The aim of this study was to describe patients' in-depth experiences of their relationship with nurses when hospitalized for anorexia and the research question was: How do women hospitalized due to anorexia nervosa experience the nursepatient relationship? The results of this study illustrate how a confident relationship with the nurse is essential, especially for those undergoing inpatient care. A relationship that enables the women to balance feelings of emotional 
contradictions will help them to cope and talk about ongoing problems. A good therapeutic relationship creates confidence and should be established as early as possible in order to encourage the patients to accept help. During the analysis we transformed the concept of treatment to the concept of care [15], where it appeared natural. A confident relationship will support the patient throughout the different phases of anorexia care.

The study illuminates how a confident relationship can be established by becoming familiar with and discovering the individual's life-world. A factor that contributes to the care outcome is the ability to become confident in the specific therapeutic method that is delivered to the patient, which highlights the need for nurses with possess knowledge and confidence. Consistency on the part of the nurse in relation to rules and efforts seems to create confidence in anorexia care. A rigid structure can support the women when it is enforced in a confident way. On the other hand, flexibility and customization of rules can also be experienced positively.

The relationship creates the basis for mercy and compassion, which allows a shared insight and understanding of anorexic problems [16]. From the beginning of the therapeutic interaction prioritization is important for creating a confident nurse-patient relationship that will not be negatively influenced by the specific treatment method. However, as underlined in this study, the patient needs a caring relationship in order to become familiar with the method. Previous research indicates that women with anorexia often have difficulty establishing a confident relationship with others, which may be related to their development and life experience [17]. Establishing a confident relationship is therefore particularly challenging.

The present study highlights the importance of predictability and individual care, which give the patient an opportunity to share difficult feelings and gain greater understanding of what influences the condition. Cohesive care is central in quality nursing that promotes health. Lindstrom [15] and Nilsson [16] focus on the importance of being available in the patient's life-world in line with her/his perceived needs. Consequently, it is vital that nurses have in-depth knowledge of establishing relationships related to different health challenges. Lindstrom [15] highlights that in order to indicate understanding, nurses should react constructively and acknowledge patient expressions. By being aware of her/his own emotions, the nurse will be able to receive, contain, and deal with the patient's feelings and reactions, which in turn can enable the patient to deal with the processed feelings constructively. If the patient triggers unprocessed feelings in the nurse's emotional life, self-knowledge enables her/him to avoid responding with unconscious negative countertransference [15].

Lindstrom [15] emphasizes the importance of striving for equality in the relationship between nurse and patient. In their research on the patient-nurse relationship, Rørtveit and Severinsson (2012) highlight the fact that nurses' leadership qualities should be associated with being formative and mindful when in a 
therapeutic process with women suffering from eating difficulties [18]. The relationship has a natural imbalance, as the nurse has more power over rules and structure, which can lead to conflict when enforced in a non-respectful way. It is therefore important to give the patient an opportunity to participate and influence decisions, thus increasing patient autonomy [15].

The present study indicates various challenges that emerge in an atmosphere of conflicting feelings. When the atmosphere is stressful due to strict rules, the caring relationship may be negatively affected. Some concrete interventions such as too much focus on weight may be perceived as a punishment, which can reduce confidence, promote negative behavior, and lessen motivation for improvement. The importance of not using treatment in a way that the patient experiences as a punishment is highlighted in previous research [19]. The skill is to use structure and rules in a supportive and motivating way to promote healthy behaviors. This is achieved by an attitude of equality and cooperation, which has been pointed out as an important nursing ability [15].

The present study reveals the benefits of individualized care and support to patients who are experiencing difficult feelings related to weight gain and the mechanisms behind the disease. Research indicates the importance of holistic care and individuality without coercion [20]. This accords with previous research, which highlights the necessity of understanding patient experiences related to weight gain [21]. Focusing solely on weight increases the risk of relapse after discharge [20]. Lindström (2003) states that nursing should be permeated by love, care, and compassion, as these attributes will guide the nursing process so that structure and rules are imposed in a supportive way that promotes health [15]. The present study does not compare the different treatment methods with reference to the patient-nurse-relationship, although previous research has revealed the importance of the relationship for treatment [15] [21]. It is through the relationship with the patient that different health promoting actions can be performed. Hence Lindström (2003) supports the participants' perception of the significance of relationships.

The theme longing for caring and responsive nurses seems to be the most significant finding of the present study and highlights the fact that anorexia care should avoid hesitancy, insecurity, and incompetence, which factors are inhibiters of confident relationship development [4] [21]. Research reveals that lack of knowledge about the disease and its impact on the patient's psychological state can result in behavior that is detrimental to the development of a therapeutic relationship [21]. The present study includes some descriptions of being mistrusted, controlled, and met with condescending attitudes, all of which are unhelpful for developing a positive relationship. Negative experiences are also related to the nurse being unavailable and lack of time. Patients welcome the nurse who assumes a sensitive and leading role as described by Rørtveit and Severinsson (2012) in their study on women with eating difficulties [18]. Aspects such as leading towards an awakening of the patients' awareness, leading in a way that 
solves the patients' existential dilemmas, addressing ambiguous feelings, and confirming ambivalence when worrying about crossing a line were described in group therapy [18]. These factors can also be seen in this theme. In addition, the study highlighted how patients were empowered by an understanding attitude and dialogues about distressing feelings such as shame [18]. These challenges require resources to ensure the establishment of a good relationship as a foundation for the treatment [16] [21]. Among others, Ackerman and Hilsenroth (2003) identified the above-mentioned factors as essential for the development of a good relationship [6]. Snell et al. (2010) emphasize that the nurse has a crucial role around the clock when caring for patients with anorexia [4]. In order to establish a good relationship it is essential that nurses have knowledge of the specific disorder and the impact of treatment methods on the patient's mental state and behavior. George (1997) likewise found that confidence, understanding, and knowledge are essential for good relationship development, and that nursing attitudes can affect the relationship and treatment outcome. A positive attitude towards patients may help to raise their self-esteem, motivation, and give them a sense of achievement [21]. When addressing the patient's life situation or "case" as Gadamer (2004) expresses it, an approach that reflects a skilled and responsive nurse who contributes confidence to the patient's life-world is important. Nurses' reflections on their own pre-understanding and attitudes also provides awareness of how their stance affects the patient [16]. This is supported by Lindström (2003), who emphasizes the importance of understanding and knowledge for obtaining a deeper meaning of the content of the patient-nurse relationship [15].

\section{Methodological Considerations}

A limitation is that only five women were interviewed for this study; however, the in-depth interviews provided rich and detailed data. The women opened up and shared their inner experiences of a vulnerable period. Their descriptions were explored by means of qualitative content analysis in order to seek understanding and reveal concealed meanings. Validity and confidence in the truth of the research process were demonstrated by remaining close to the women's voices, which were represented by the quotations. However, the findings in qualitative studies are unlikely to be transferable due to the small number of participants. This study can only be validated by confirmation from other with similar experiences. The women were recruited from a small area of Norway but had experiences of different in-patient environments. While a description of these environments could strengthen the study, it was not included as we had no data on the actual time and place in question.

\section{Conclusions and Implications}

The present study revealed that a confident relationship and care help patients to achieve their goals and manage difficult situations. It demonstrates the impor- 
tance of an individual focus, which is valuable when helping those suffering from anorexia to deal with negative emotions and simultaneously working with weight gain. When undergoing anorexia care it is essential that patients are cared for, receive help, and feel confident from the start.

\section{Implications for Practice and Research}

Knowledge from this study should be used to ensure that patients receive help to cope with their existence in the world and experience quality of life. In the process of approaching patients with anorexia, the nurse-patient relationship has a crucial impact on quality of care.

A structured treatment process is perceived to be effective, but relies on the establishment of a confident relationship. The specific treatment method will not hinder the development of a positive relationship if the relationship is established on the basis of the necessary nursing attitudes and characteristics described in this study.

It is important that nurses are familiar with and have professional skills related to factors that affect the development of a positive relationship. Reflection and insight into the patient's personal lifeworld are required when treating patients with anorexia. This knowledge and increased understanding may help to highlight the significance of care relationships and the importance of prioritizing education for nurses of patients with anorexia.

Education and nursing supervision will increase knowledge of the significance of relationships, methods, and an ethical attitude in nursing practice. This knowledge may be useful in hospital wards, but might also promote good anorexia care in primary care for this group of patients.

It is possible that the development of a structured hermeneutic nursing intervention for patients suffering from anorexia may contribute as a tool that improves the quality of care. The intervention will enable meetings to be arranged where patients suffering from anorexia are encountered in their life world, leading to the establishment of a confident relationship as a basis for high quality care. A hermeneutic nursing approach can help the nurse and patient to work together with structure and rules in the patient's lifeworld, while allowing the nurse to gain a deeper understanding of the patient's mental state.

Documented research is essential when advancing scientific knowledge and ensuring qualified care for people who are suffering in various areas of their life world. A carefully considered research process and analysis will make it possible to further develop and provide new insights into relationship issues and care of hospitalized adult anorexia patients.

\section{Acknowledgements}

We would like to thank the women who shared their innermost experiences in order to provide data for this study. We would also like to express our thanks to the University Hospital of Stavanger for financial support and to Monique Fe- 
dersel for reviewing the English language. We acknowledge that the study was supported by grants from Stavanger University Hospital, Stavanger, Norway.

\section{Authors' Contributions}

KR was responsible for writing the manuscript. AM was responsible for the data collection. All authors contributed to the critical revision of the intellectual content, provided feedback on the draft manuscript and approved the final version. They all adhered to the criteria pertaining to roles and responsibilities in the research process recommended by the International Committee of Medical Journal Editors (ICMJE) (http://www.icmje.org/recommendations).

\section{Conflicts of Interest}

The authors declare no conflicts of interest regarding the publication of this paper.

\section{References}

[1] Malt, U. and Aslaksen, P. (2020) Psykiske Lidelser. I Store Medisinske Leksikon. https://sml.snl.no/psykiske_lidelser

[2] Fairburn, C.G. and Harrison, P.J. (2003) Eating Disorders. The Lancet, 361, 407416. https://doi.org/10.1111/j.1365-2850.2011.01760.x

[3] Wright, K.M. and Hacking, S. (2012) An Angel on My Shoulder: A Study of Relationships between Women with Anorexia and Healthcare Professionals. Journal of Psychiatric and Mental Health Nursing. 19, 107-115. https://doi.org/10.1111/j.1365-2850.2011.01760.x

[4] Snell, L., Crowe, M. and Jordan, J. (2010) Maintaining a Therapeutic Connection: Nursing in an Inpatient Eating Disorder Unit. Journal of Clinical Nursing, 19, 351358. https://doi.org/10.1111/j.1365-2702.2009.03000.x

[5] Zugai, J.S., Stein-Parbury, J. and Roche, M. (2018) The Nature of the Therapeutic Alliance between Nurses and Consumers with Anorexia Nervosa in the Inpatient Setting: A Mixed-Methods Study. Journal of Clinical Nursing, 27, 416-426. https://doi.org/10.1111/jocn.13944

[6] Ackerman, S.J. and Hilsenroth, M.J. (2003) A Review of Therapist Characteristics and Techniques Positively Impacting the Therapeutic Alliance. Clinical Psychology Review, 23, 1-33. https://doi.org/10.1016/S0272-7358(02)00146-0

[7] Swatton, A. (2011) Transference and Countertransference in Anorexia Nervosa Care. Gastrointestinal Nursing, 9, 38-43. https://doi.org/10.12968/gasn.2011.9.3.38

[8] Raeve, L. (2002) Trust and Trustworthiness in Nurse-Patient Relationship. Nursing Philosophy, 3, 152-162. https://doi.org/10.1046/j.1466-769X.2002.00090.x

[9] Waller, G., Evans, J. and Stringer, H. (2012) The Therapeutic Alliance in the Early Part of Cognitive-Behavioral Therapy for the Eating Disorders. International Journal of Eating Disorders, 45, 63-69. https://doi.org/10.1002/eat.20914

[10] Gadamer, H.-G. (2004) Truth and Method. Continuum, London.

[11] Gadamer, H.-G. (1989) Truth and Method. In: Weinsheimer, J. and Marshall, D.G., Ed., Wahrheit Und Methode, 2nd Edition, Sheed\& Ward, London.

[12] Rortveit, K., Astrom, S. and Severinsson, E. (2010) The Meaning of Guilt and Shame: A Qualitative Study of Mothers Who Suffer from Eating Difficulties. International 
Journal of Mental Health Nursing, 19, 231-239.

https://doi.org/10.1111/j.1447-0349.2010.00672.x

[13] Graneheim, U.H. and Lundman, B. (2004) Qualitative Content Analysis in Nursing Research: Concepts, Procedures and Measures to Achieve Trustworthiness. Nurse Education Today, 24, 105-112. https://doi.org/10.1016/j.nedt.2003.10.001

[14] WMA (2008) World Medical Association Assembly.

[15] Lindström, U.Å. (2003) Psykiatrisk Sykepleie: Teori, Verdier Og Praksis (Psychiatric Nursing: Theory, Values and Praxsis). Gyldendal Akademisk, Oslo, $160 \mathrm{~s}$.

[16] Nilsson, K. (2007) Recovery from Adolescent Onset Anorexia Nervosa-A Longitudinal Study. Umeå University Medical Dissertations, New Series No. 1098, Umeå University, Umeå.

[17] De La Rie, S., Noordenbos, G., Donker, M. and Van Furth, E. (2008) The Quality of Treatment of Eating Disorders: A Comparison of the Therapists' and the Patients' Perspective. International Journal of Eating Disorders, 41, 307-317.

https://doi.org/10.1002/eat.20494

[18] Rørtveit, K. and Severinsson, E. (2012) Leadership Qualities When Providing Therapy for Women Who Suffer from Eating Difficulties. Journal of Nursing Management, 20, 631-639.

[19] Colton, P., et al. (2004) Disturbed Eating Behavior and Eating Disorders in Preteen and Early Teenage Girls with Type 1 Diabetes: A Case-Controlled Study. Diabetes Care, 27, 1654-1659. https://doi.org/10.2337/diacare.27.7.1654

[20] Escobar-Koch, T., et al. (2010) Service Users' Views of Eating Disorder Services: an International Comparison. International Journal of Eating Disorders, 43, 549-559. https://doi.org/10.1002/eat.20741

[21] George, L. (1997) The Psychological Characteristics of Patients Suffering from Anorexia Nervosa and the Nurse's Role in Creating a Therapeutic Relationship. Journal of Advanced Nursing, 26, 899-908.

https://doi.org/10.1046/j.1365-2648.1997.00421.x 